\title{
Dispersive quantum systems
}

\section{A class of isolated non-time reversal invariant quantum systems}

\author{
Lúcio Fassarella
}

September-01/2011

\begin{abstract}
A dispersive quantum system is a quantum system which is both isolated and non-time reversal invariant. This article presents precise definitions for those concepts and also a characterization of dispersive quantum systems within the class of completely positive Markovian quantum systems in finite dimension (through a homogeneous linear equation for the non-Hamiltonian part of the system's Liouvillian). To set the framework, the basic features of quantum mechanics are reviewed focusing on time evolution and also on the theory of completely positive Markovian quantum systems, including Kossakowski-Lindblad's standard form for Liouvillians. After those general considerations, I present a simple example of dispersive two-level quantum system and apply that to describe neutrino oscillation.
\end{abstract}

Keywords quantum time evolution · non-time reversal invariance $\cdot$ isolated quantum system $\cdot$ neutrino oscillation

PACS 03.65.Aa $\cdot 03.65 . \mathrm{Yz} \cdot 13.15 .+\mathrm{g}$

\section{Introduction}

The search for a scientific understanding of time has a wide scope in physics, ranging from classical mechanics to quantum field theory, from particle mechanics to cosmology, statistical physics and beyond. The research on time inevitably touches foundational issues and one can even suspect it cannot be fully understood since time is so essential to our perception of reality. Nevertheless, we can hope to improve our knowledge about time as time goes on...

Universidade Federal do Espírito Santo

CEUNES - Rodovia BR 101 Norte, Km. 60, CEP 29932-540, São Mateus - ES, Brazil

Tel.: $+55(27) 3312-1511$

Fax: $+55(27) 3312-1510$

E-mail: lucio.fassarella@ufes.br 
Instead of to deal with the subtleties of the physical meaning of time (for detailed discussion see [1] and [2] and references quoted therein), here I'm devoted to a simpler task: to show that it is theoretically possible an elementary quantum system be both isolated and non-time reversal invariant. This possibility contradicts a common sense among physicists, namely, that isolated systems are (ever) time reversal invariant and that irreversibility is just a statistical phenomenon (coded in the Second Law of Thermodynamics).

To be more precise, consider a quantum system and denote its state (density operator) in time $t$ by $\rho(t)$ - here, I use Schrödinger's picture. It is generally accepted that if the system is closed, then its time evolution is given by von Neumann's equation with some time-dependent Hamiltonian $H(t)$ :

$$
\frac{d}{d t} \rho(t)=-\frac{i}{\hbar}[H(t), \rho(t)]
$$

Accordingly, the system is said to be isolated when it is closed and its Hamiltonian is constant. So, according with this view open quantum systems are exactly those whose time evolution is not governed by (time-dependent) von Neumann equation (1). In books and papers those conceptions may be used implicitly; for example, authors can relate the semigroup structure of time evolution to (Markovian) open systems only ${ }_{1}^{1}$

Although the above definitions for the concepts of isolated, closed and open can be mathematically perfect, they lack direct correspondence with those physical meanings we give to them. Indeed, when we say a physical system is closed we mean that it does not exchange matter with other systems; also, we say a physical system is isolated when it does not interact with any other system. In this sense, one realizes that the concept of isolated system must be related to the Principle of Inertia, meaning that after arbitrary preparation, an isolated system has its energy and momenta remaining constant. Assuming the operationalist point of view, I define the concept of isolated quantum systems in terms of the expectation values of its energy-momentum tensor operator:

Definition 1 A quantum system is isolated when the expectation values of its energymomentum tensor operator with respect to any time-dependent state is constant.

I argue that this definition is not trivially irrelevant, even though it is a truism that there is no isolated system (within Universe) - at least, they could not be observed even if they existed $\left.\right|^{2}$ Nevertheless, many systems can be regarded isolated in practice, at least for a short interval of time, and this is sufficient to the concept be (eventually) useful.

\footnotetext{
1 There are many examples, but I mention only the book by Breuer-Petruccione [3] p.110], the article by G.E. Crooks [4] and some pioneers on the subject: V. Gorini at al. [5], G. Lindblad [6], Mehra-Sudarshan [7], Kossakowski [8] and Ingarden-Kossakowski [9].

2 According to Breuer-Petrucione [3 p.vii]: "Quantum mechanical-systems must be regarded as open systems. On the one hand, this is due to the fact that, like in classical physics, any realistic system is subjected to a coupling to an uncontrollable environment which influences it in a non-negligible way. The theory of open quantum systems thus plays a major role in many applications of quantum physics since perfect isolation of quantum systems is not possible and since a complete microscopic description or control of the environmental degrees of freedom is not feasible or only partially so."
} 
Beyond the almost triviality of give a definition for the concept of isolated quantum system, I'm going to highlight a special class of them, namely, isolated quantum systems that are non-time reversal invariant ${ }^{3}$ Quantum systems of this sort I call dispersive quantum systems:

Definition 2 A dispersive quantum system is a quantum system which is isolated and non-time reversal invariant.

Perhaps, a distinguished example of dispersive quantum system is the whole Universe ${ }^{4}$ However, it would be remarkable if we could discover a dispersive quantum system being also elementary, because its non-time reversal invariance would be fundamental - in the sense of not being an emergent (statistical) property. The dispersive qubit (section 3) and its application to neutrino oscillation (section 4) are intended to instantiate that!

The structure of the paper is simple. Section 2 starts with general quantum mechanics focusing on time evolution and a definition of irreversibility, followed by a review of definitions and results about Markovian quantum systems, dynamical semigroups and completely positiveness. After, it is obtained the equation for the Liouvillian's non-Hamiltonian part that characterizes those completely positive Markovian quantum systems that are isolated. In section 3, it is presented an explicit model exhibiting the mentioned features. In section 4 I apply previous developments to describe neutrino oscillation, with the introduction of a new parameter I call dispersive parameter. In the final section 5, some remarks are discussed.

Remark 1 I denote by $\mathbb{N}$ the set of natural numbers including zero and set $\mathbb{N}^{*}:=$ $\mathbb{N} \backslash\{0\}$. For a Hilbert space $\mathscr{H}$, I denote by $\mathscr{L}(\mathscr{H})$ the set of densely defined operators in $\mathscr{H}$, by $\mathscr{B}(\mathscr{H})$ the space of bounded operators in $\mathscr{H}$ and by $\mathscr{T}(\mathscr{H})$ the space of bounded trace class operators in $\mathscr{H}$. Finally, I use natural units, so $\hbar=1$.

\section{Quantum mechanical systems}

\subsection{Basic structure}

In Quantum Mechanics, physical systems are described in terms of observables and states with the use of a separable Hilbert space $\mathscr{H}$ : for a system $\mathfrak{S}$ without superselection sectors, observables are identified with (densely defined) self-adjoint operators in $\mathscr{H}$ and states are identified with density operators, i.e., positive trace class operators with trace one in $\mathscr{H}$. I denote the set of observables by $\mathscr{A}(\mathscr{H})$ and the set of states by $\mathscr{S}(\mathscr{H})$. The fundamental postulate of Quantum Mechanics states that the expectation value of an observable $A \in \mathscr{A}(\mathscr{H})$ when the system is in the state

\footnotetext{
3 Non-time reversal invariance is defined in section 2

4 Actually, in order to the Universe be consistently regarded isolated we must take gravity into account, as it was noted by Landau and Lifschitz in [12. p.30]; otherwise, we should describe matter as under the influence of ever changing external conditions.
} 
$\rho \in \mathscr{S}(\mathscr{H})$ is given by

$$
\langle A \mid \rho\rangle:=\operatorname{tr}(\rho A)
$$

Time evolution. Consider the system was prepared at time $t_{0}$ and evolves without interference since then. In Schrödinger's picture, system's time evolution from an instant $t_{1} \geq t_{0}$ to a later instant $t_{2}$ must be described by a map from the space of states to itself,

$$
\Gamma_{t_{2}, t_{1}}: \mathscr{S}(\mathscr{H}) \rightarrow \mathscr{S}(\mathscr{H})\left(t_{2}>t_{1}\right)
$$

I call this time evolution map and its physical interpretation is simple: given two in stants $t_{2}>t_{1} \geq t_{0}$, if $\rho_{1}$ is the system's state at instant $t_{1}$, then $\Gamma_{t_{2}, t_{1}} \rho_{1}$ is the system's state at the instant $t_{2}>t_{1}$. It is natural to assume the following property I call factorization:

$$
\Gamma_{t_{3}, t_{1}}=\Gamma_{t_{3}, t_{2}} \Gamma_{t_{2}, t_{1}}, \forall t_{3}>t_{2}>t_{1} \geq t_{0}
$$

A physical system is time reversal invarian ${ }^{6}$ when there is a map $\Upsilon: \mathscr{S}(\mathscr{H}) \rightarrow$ $\mathscr{S}(\mathscr{H})$ satisfying

i) Idempotence:

$$
\Upsilon^{2}=i d
$$

ii) Time-reversing equation:

$$
\Gamma_{t_{2}, t_{1}} \Upsilon \Gamma_{t_{2}, t_{1}}=\Upsilon \quad, \forall t_{2}>t_{1} \geq t_{0}
$$

I call $\Upsilon$ the time reversing map. By physical reasons, one may require additional properties on $\Upsilon$, such as antilinearity. Its physical meaning is natural: $\Upsilon$ defines a correspondence among the system's states which reverts the direction of time evolution:

$$
\Upsilon \Gamma_{t_{2}, t_{1}} \Upsilon=\Gamma_{t_{2}, t_{1}}^{-1}, \forall t_{2}>t_{1} \geq t_{0}
$$

This equation is the quantum analog of what can in principle be done to a classical (non-magnetic) system: if the velocities of all particles of a classical mechanical system are reversed (what can be represented by a map in phase space), then this system would behave as if it was running backwards in time 7

A system is non-time reversal invariant when it is not time reversal invariant. Time reversal invariance holds for some physical systems, but one does not have any reason to assume this property must hold for all closed physical systems. (Actually, I'm going to describe a class of isolated non-time reversal invariant quantum systems.)

\footnotetext{
5 There are some technical requirements to this formula to be well defined in general, but it is always well defined when $A$ is bounded - what is automatic in the finite dimensional case, which is the one we are interested.

6 Time reversal invariance is a property of systems, while reversibility is a property of a system's states. In general, the non-time reversal invariance of a system is related to the existence of an irreversible state. See [10] and [11] for a more detailed discussion on the concepts of time reversal invariance and reversibility, as well as the relation between them and the Second Law of Thermodynamics.

7 In general, isolated classical systems are time reversal invariant; but for a system with very large number of degrees of freedom, its macroscopic behavior exhibits statistical properties that allow us to distinguish the past from the future.
} 
If the system is time reversal invariant, equations (5) and (6) imply $\Gamma_{t_{2}, t_{1}}$ is invertible, for all $t_{2}>t_{1} \geq t_{0}-$ so, time-evolution map's invertibility is a necessary condition for the system to be invertible:

$$
\Gamma_{t_{2}, t_{1}}^{-1}=\Upsilon \Gamma_{t_{2}, t_{1}} \Upsilon, \forall t_{2}>t_{1} \geq t_{0}
$$

In this case, one can define the extended time evolution map in $\mathscr{S}(\mathscr{H})$ :

$$
\tilde{\Gamma}_{t_{2}, t_{1}}: \mathscr{S}(\mathscr{H}) \rightarrow \mathscr{S}(\mathscr{H}) \quad, \quad \tilde{\Gamma}_{t_{2}, t_{1}}:=\left\{\begin{array}{c}
\Gamma_{t_{2}, t_{1},}, t_{2}>t_{1} \geq t_{0} \\
i d, t_{2}=t_{1} \geq t_{0} \\
\Gamma_{t_{2}, t_{1}}^{-1}, t_{1}>t_{2} \geq t_{0}
\end{array} \quad, \forall t_{1}, t_{2} \geq t_{0}\right.
$$

The extended time evolution map satisfies extended factorization:

$$
\tilde{\Gamma}_{t_{3}, t_{1}}=\tilde{\Gamma}_{t_{3}, t_{2}} \tilde{\Gamma}_{t_{2}, t_{1}}, \forall t_{3}, t_{2}, t_{1} \geq t_{0}
$$

Proof: I have to analyze the six possible orderings for instants $t_{1}, t_{2}, t_{3} \geq t_{0}$; the case $t_{3}>t_{2}>t_{1} \geq t_{0}$ follows directly from factorization (4); here, I verify explicitly the case $t_{2}>t_{3}>t_{1} \geq t_{0}$ only:

$$
\tilde{\Gamma}_{t_{3}, t_{1}}=\Gamma_{t_{1} t_{3}}=\Gamma_{t_{2}, t_{3}}^{-1} \Gamma_{t_{2}, t_{3}} \Gamma_{t_{3}, t_{1}}=\Gamma_{t_{2}, t_{3}}^{-1} \Gamma_{t_{2}, t_{1}}=\tilde{\Gamma}_{t_{3}, t_{2},} \tilde{\Gamma}_{t_{2}, t_{1}}
$$

Remark 2 For a time reversal invariant system, there is no intrinsic distinction between past and future, since any pair of states related by time evolution map are equally related by the time evolution map's inverse. However, an observer assigns a "time arrow" to an non-time reversal invariant system through preparation time $t_{0}$ : before $t_{0}$ the system interacts with environment and after $t_{0}$ the system evolves without external interaction [12, p.32], [13, §7].

Markovian systems. A system is said to be Markovian when its time evolution map depends of the time interval between instants only:

$$
\Gamma_{t_{2}, t_{1}}=\Gamma_{t_{0}+t_{2}-t_{1}, t_{0}}, \forall t_{2}>t_{1} \geq t_{0}
$$

In this case, the system's time evolution is given by the (one-parameter) quantum dynamical semigroup in $\mathscr{S}(\mathscr{H})$ with domain $[0, \infty)$ :

$$
\Gamma_{t}: \mathscr{S}(\mathscr{H}) \rightarrow \mathscr{S}(\mathscr{H}), \Gamma_{t}:= \begin{cases}i d & , t=0 \\ \Gamma_{t_{0}+t, t_{0}}, & t>0\end{cases}
$$

Directly from factorization (4), it follows the semigroup property:

$$
\Gamma_{t_{2}} \Gamma_{t_{1}}=\Gamma_{t_{2}+t_{1}}, \forall t_{1}, t_{2} \geq 0
$$

Proof: $\Gamma_{t_{2}} \Gamma_{t_{1}}=\Gamma_{t_{2}+t_{1}, t_{1}} \Gamma_{t_{1}, 0}=\Gamma_{t_{2}+t_{1}, 0}=\Gamma_{t_{2}+t_{1}}, \forall t_{1}, t_{2}>0$.

Remark 3 Physically, time evolution is Markovian when it does not depend on the past (or future) history of the quantum system and its environment - there is no "memory" about the way it reaches its present state. As far as I know, Markovian property holds for closed systems and, as it was demonstrated by Davies in [14], it holds also for open systems under special conditions. 
If the system is Markovian and time reversal invariant, its quantum dynamical semigroup can be extended to a one-parameter group in $\mathscr{S}(\mathscr{H})$, with its group property being a consequence of the semigroup property (12):

$$
\tilde{\Gamma}_{t}: \mathscr{S}(\mathscr{H}) \rightarrow \mathscr{S}(\mathscr{H}) \quad, \tilde{\Gamma}_{t}:=\left\{\begin{array}{cc}
\Gamma_{t}, t \geq 0 \\
\Gamma_{-t}^{-1}, t<0
\end{array}\right.
$$

Quantum dynamical semigroup's generator. For technical reasons (to appear in subsection 2.2), from now on assume the quantum dynamical semigroup can be extended to a semigroup of positive trace preserving superoperator 8 in the space $\mathscr{T}(\mathscr{H})$ of bounded trace class operators in $\mathscr{H}$,

$$
\Gamma_{t}: \mathscr{T}(\mathscr{H}) \rightarrow \mathscr{T}(\mathscr{H}), t \geq 0
$$

Semigroup property:

$$
\Gamma_{t_{2}+t_{1}}=\Gamma_{t_{2}} \Gamma_{t_{1}}, \forall t_{2}, t_{1} \geq 0
$$

Positivity:

$$
\Gamma_{t}\left(\sigma^{*} \sigma\right) \geq 0, \forall \sigma \in \mathscr{T}(\mathscr{H})
$$

Trace preserving property:

$$
\operatorname{tr}\left(\Gamma_{t}(\sigma)\right)=\operatorname{tr}(\sigma), \forall \sigma \in \mathscr{T}(\mathscr{H})
$$

Also, one assume the quantum dynamical semigroup is $\|\cdot\|_{1}$-continuous from above:

$$
\lim _{t \downarrow 0}\left\|\Gamma_{t}(\sigma)-\sigma\right\|_{1}=0, \forall \sigma \in \mathscr{T}(\mathscr{H})
$$

The space $\mathscr{T}(\mathscr{H})$ of trace class operators in $\mathscr{H}$ is a Banach space w.r.t. the trace-norm $\|\cdot\|_{1}$

$$
\|\sigma\|_{1}:=\operatorname{tr} \sqrt{\sigma^{*} \sigma}, \forall \sigma \in \mathscr{T}(\mathscr{H})
$$

Moreover, $\mathscr{T}(\mathscr{H}) \subset \mathscr{B}(\mathscr{H})$ (trace class operators are bounded) and the space of finite-rank operators in $\mathscr{H}$ is $\|\cdot\|_{1}$-dense in $\mathscr{T}(\mathscr{H})$. For details, see [21, pp.206209] 9

The above conditions are sufficient to guarantee that the quantum dynamical semigroup has an infinitesimal generator:

\footnotetext{
8 The term superoperator is used for operators in a space of operators.

9 Note that the notation of [21] differs from our notation: in [21], $\mathscr{L}(\mathscr{H})$ denotes the space of bounded operators in $\mathscr{H}$, but here it denotes space of densely defined operators in $\mathscr{H}$.
} 
Theorem 1 (quantum dynamical semigroup's generator) For the quantum dynamical semigroup (12) under conditions (16), (17), (18) and (19), it holds:

i) There exists an operator $L$ with $\|\cdot\|_{1}$-dense domain $\operatorname{Dom}(L) \subset \mathscr{T}(\mathscr{H})$ such that

$$
\lim _{t \downarrow 0}\left\|\frac{\Gamma_{t}(\sigma)-\sigma}{t}-L(\sigma)\right\|_{1}=0, \forall \sigma \in \operatorname{Dom}(L)
$$

ii) The quantum dynamical semigroup $\Gamma_{t \geq 0}$ is a limit of exponentials related to $L$ :

$$
\Gamma_{t}(\sigma)=\lim _{\varepsilon \downarrow 0}\left(\exp \left(t L_{\varepsilon}\right) \sigma\right), \forall \sigma \in \operatorname{Dom}(L), \forall t \geq 0
$$

where

$$
L_{\varepsilon}:=\frac{\Gamma_{\varepsilon}-i d}{\varepsilon}, \forall \varepsilon>0
$$

iii) $L$ is bounded if and only if $\operatorname{Dom}(L)=\mathscr{T}(\mathscr{H})$. In this case, $\Gamma_{t \geq 0}$ is the exponential of $L$ :

$$
\Gamma_{t}(\sigma)=\exp (t L) \sigma, \forall \sigma \in \mathscr{T}(\mathscr{H}), \forall t \geq 0
$$

Further, in this situation it holds

$$
\lim _{t \downarrow 0}\left\|\Gamma_{t}-i d\right\|_{1}=0
$$

In Quantum Mechanics, the generator L of the system's quantum dynamical semigroup is called the system's Liouvillian superoperator.

For a proof, see [22, pp.376-379] or [23, p.237-238]. The limit 21] combined with the fact that the operator norm and trace-norm satisfy [21, p.209]

$$
\|\sigma\| \leq\|\sigma\|_{1}, \forall \sigma \in \mathscr{T}(\mathscr{H})
$$

implies the system's equation of motion, called Markovian quantum master equation [3, p.119]:

$$
\frac{d}{d t} \rho(t)=L(\rho(t))
$$

Remark 4 From the mathematical point of view, Theorem (1) generalizes Stone's Theorem, which establishes the existence and uniqueness of the infinitesimal generator for an one-parameter group of unitary operators in Hilbert spaces - for details see [22, Theorem 13.38, p.382]. From the physical point of view, Theorem (1) generalizes for completely positive Markovian quantum systems the von Neumann equation for time-dependent states (1).

Remark 5 The Hille-Yosida Theorem characterizes those operators in $\mathscr{H}$ which are generators of some dynamical semigroup [22, Theorem 13.37, p.380], [23, pp.246249]: a densely defined operator $L$ in a Banach space $X$ is the generator of some trace-norm continuous one-parameter semigroup of bounded operator in $X$ if and only if there are constants $C>0$ and $\gamma \in \mathbb{R}$ such that

$$
\left\|(\zeta I-L)^{-m}\right\|_{\mathscr{B}(X)} \leq C(\zeta-\gamma)^{-m}, \forall \zeta>\gamma, \forall m \in \mathbb{N}^{*}
$$

where \|\|$_{\mathscr{B}(X)}$ is the operator norm in $\mathscr{B}(X)$. 
It is natural to ask about the general form of the Liouvillian of a Markovian quantum system. Fortunately, this question has an answer for the special class of quantum dynamical semigroups which are completely positive. This is the point of the following subsection.

2.2 Completely positive quantum dynamical semigroups

Completely positiveness (see Appendix A is a property of quantum operations, a concept of the theory of generalized measurements [3, pp.85-89], [15]. This is a special property of a large class of quantum systems, including subsystems of systems whose time evolution is unitary and do satisfy some special conditions [3, pp.122123] [16]. Such systems have a wide range of applications, ranging from quantum information theory [17] to astrophysics [18] [19] (to give only two examples).

If the Liouvillian of a completely positive quantum dynamical semigroup is bounded, then it has a standard form, according to the following Theorems due to Lindblad [6] (bounded Liouvillian) and also Gorini-Kossakowski-Sudarshan [5] (finite dimensional Hilbert space). Although the finite dimensional case can be viewed as a special case of the general Theorem due to Lindblad, it can be better scrutinized; so, I will state them separately. As far as I know, those theorems have not been extended to the case of unbounded Liouvillians, a typical situation in physics.

Theorem 2 (Standard form of a bounded Liouvillian [6]) Let L be the Liouvillian of the quantum dynamical semigroup $\Gamma_{t}>0$ of a completely positive Markovian quantum system $\mathfrak{S}$ with Hilbert space $\mathscr{H}$.

If $L$ is bounded (equivalently, $\operatorname{Dom}(L)=\mathscr{T}(\mathscr{H})$ ), then there exist a bounded selfadjoint operator $H$ in $\mathscr{H}$ and a countable family of bounded operators $V_{1}, V_{2}, \ldots$ in $\mathscr{H}$ satisfying

$$
\sum_{j} V_{j}^{*} V_{j} \in \mathscr{B}(\mathscr{H})
$$

such that

$$
L(\sigma)=-i[H, \sigma]+\frac{1}{2} \sum_{j}\left(V_{j} \sigma V_{j}^{*}-\frac{1}{2}\left(V_{j}^{*} V_{j} \sigma+\sigma V_{j}^{*} V_{j}\right)\right), \forall \sigma \in \mathscr{T}(\mathscr{H})
$$

Theorem 3 (Standard Form of a Liouvillian in a finite dimensional Hilbert [5]) Let $L$ be the Liouvillian of the quantum dynamical semigroup $\Gamma_{t \geq 0}$ of a completely positive Markovian quantum system $\mathfrak{S}$ with Hilbert space $\mathscr{H}$ having finite dimension $N=\operatorname{dim} \mathscr{H}$. In this case $\mathscr{T}(\mathscr{H})=\mathscr{B}(\mathscr{H})=\mathscr{L}(\mathscr{H})$ has dimension $N^{2}$.

Let $\left(F_{j}\right)_{j=1 \ldots N^{2}}$ be a complete set in $\mathscr{B}(\mathscr{H})$ with $F_{N^{2}}=I / \sqrt{N}$ which is orthonormal w.r.t. trace, i.e.,

$$
\operatorname{tr}\left(F_{i}^{*} F_{j}\right)=\delta_{i j}, \forall i, j=1, \ldots, N^{2}
$$

Then, there exist a self-adjoint operator $H$ in $\mathscr{H}$ and a unique positive complex matrix $\left(a_{i j}\right) \in M_{N^{2}-1}(\mathbb{C})$ such that

$$
L(\sigma)=-i[H, \sigma]+\sum_{i, j=1}^{N^{2}-1} a_{i j}\left(F_{i} \sigma F_{j}^{*}-\frac{1}{2}\left(F_{j}^{*} F_{i} \sigma+\sigma F_{j}^{*} F_{i}\right)\right), \forall \sigma \in \mathscr{T}(\mathscr{H})
$$


Further, the matrix $\left(a_{i j}\right)$ is unique for each family $\left(F_{j}\right)$ and the operator $H$ is unique if it is required $\operatorname{tr}(H)=0$.

Remark 6 The conditions on $\left(F_{j}\right)_{j=1}^{N^{2}}$ in Theorem 3 imply that all operators different from $F_{N^{2}}$ are traceless

$$
\operatorname{tr}\left(F_{j}\right)=0, \forall j=1, \ldots, N^{2}-1
$$

Remark 7 Expression (32) of Theorem 3 can be transformed in the expression 30 of Theorem 2 by a diagonalization of the positive-semidefinite matrix $\left(a_{i j}\right)$, as done in [5] and [3, pp.121-122].

The operator $H$ is called Hamiltonian and the term $-i[H,$.$] is called the Hamilto-$ nian part of the system's Liouvillian.The dissipator superoperator is defined by (in agreement with [3, p.123]):

$$
\mathscr{D}(\sigma):=L(\sigma)+i[H, \sigma]
$$

In the finite dimensional case,

$$
\mathscr{D}(\sigma)=\sum_{i, j=1}^{N^{2}-1} a_{i j}\left(F_{i} \sigma F_{j}^{*}-\frac{1}{2}\left(F_{j}^{*} F_{i} \sigma+\sigma F_{j}^{*} F_{i}\right)\right)
$$

People call $\left(a_{i j}\right)$ the system's Kossakowski matrix and note that it has dimension $N^{2}-1$ when the Hilbert space has finite dimension $N$.

\subsection{Hamiltonian's expectation value}

In this subsection I analyze Hamiltonian' expectation value values related to timedependent states for completely positive Markovian quantum systems. Our considerations are restricted to the finite dimensional case, so $\mathscr{T}(\mathscr{H})=\mathscr{B}(\mathscr{H})=\mathscr{L}(\mathscr{H})$ and the system's Liouvillian is given by Lindblad's form 32.

Definition 3 (Dissipation Operator) Consider a quantum system $\mathfrak{S}$ with Hamiltonian $H$ and dissipator superoperator having the form 33 . The system's dissipation operator is defined by the action of dissipator superoperator on the Hamiltonian:

$$
D_{H}:=\mathscr{D}(H)=\sum_{i, j=1}^{N^{2}-1} a_{i j}\left(F_{j}^{*} H F_{i}-\frac{1}{2} F_{j}^{*} F_{i} H-\frac{1}{2} H F_{j}^{*} F_{i}\right)
$$

Proposition 1 Let $\rho(t)$ be a time-dependent state of the system $\mathfrak{S}$. Then the related time-dependent Hamiltonian's expectation value value satisfies the equation

$$
\frac{d}{d t}\langle H \mid \rho(t)\rangle=\operatorname{tr}\left\{\rho(t) D_{H}\right\}
$$

${ }^{10}$ Proof: $\operatorname{tr}\left(F_{j}\right)=\operatorname{tr}\left(I F_{j}\right)=\sqrt{N} \operatorname{tr}\left(F_{N^{2}}^{*} F_{j}\right)=0, \forall j \in\left\{1, \ldots, N^{2}-1\right\}$ 
Proof The Markovian quantum master equation (27) with Liouvillian having the form (32) reads

$$
\frac{d}{d t} \rho(t)=-i[H, \rho(t)]+\mathscr{D}(\rho(t))
$$

Since derivation commutes with trace, it holds

$\frac{d}{d t}\langle H \mid \rho(t)\rangle=\frac{d}{d t} \operatorname{tr}\{H \rho(t)\}=\operatorname{tr}\left\{H \frac{d}{d t} \rho(t)\right\}=-\operatorname{itr}\{H[H, \rho(t)]\}+\operatorname{tr}\{H \mathscr{D}(\rho(t))\}$

Using that trace is invariant under cyclic permutation of factors, it follows

$\operatorname{tr}\{H[H, \rho(t)]\}=\operatorname{tr}\{H H \rho(t)-H \rho(t) H\}=\operatorname{tr}\{H H \rho(t)-H H \rho(t)\}=\operatorname{tr}\{0\}=0$

Therefore

$$
\frac{d}{d t}\langle H \mid \rho(t)\rangle=\operatorname{tr}\{H \mathscr{D}(\rho(t))\}=\operatorname{tr}\{\mathscr{D}(\rho(t)) H\}
$$

Using (32) to expand the expression inside trace, it follows

$$
\begin{aligned}
\frac{d}{d t}\langle H \mid \rho(t)\rangle & =\operatorname{tr}\{\mathscr{D}(\rho(t)) H\} \\
& =\sum_{i, j=1}^{N^{2}-1} a_{i j} t r\left\{F_{i} \rho(t) F_{j}^{*} H-\frac{1}{2} F_{j}^{*} F_{i} \rho(t) H-\frac{1}{2} \rho(t) F_{j}^{*} F_{i} H\right\} \\
& =\sum_{i, j=1}^{N^{2}-1} a_{i j} t r\left\{\rho(t) F_{j}^{*} H F_{i}-\frac{1}{2} \rho(t) H F_{j}^{*} F_{i}-\frac{1}{2} \rho(t) F_{j}^{*} F_{i} H\right\} \\
& =\operatorname{tr}\left\{\rho(t) \sum_{i, j=1}^{N^{2}-1} a_{i j}\left(F_{j}^{*} H F_{i}-\frac{1}{2} F_{j}^{*} F_{i} H-\frac{1}{2} H F_{j}^{*} F_{i}\right)\right\} \\
& =\operatorname{tr}\left\{\rho(t) D_{H}\right\}
\end{aligned}
$$

Corollary 1 The Hamiltonian's expectation value value is constant for whatever be the system's time-dependent state if and only if the system's dissipation operator is identically zero.

Proof From Proposition 1] the Hamiltonian's expectation value value is constant whatever be the system's time-dependent state if and only if $\operatorname{tr}\left\{\rho D_{H}\right\}=0 \forall \rho \in$ $\mathscr{S}(\mathscr{H})$; this is equivalent to $\left\langle D_{H} \phi \mid \phi\right\rangle=0 \forall \phi \in \mathscr{H}$ and this is equivalent to $D_{H}=$ 0 .

The above result motivates the following definition:

Definition 4 (Markovian Dispersive Quantum System) A Markovian dispersive quantum system is a Markovian quantum system whose dissipation operator is identically zero. 
Remark 8 In the finite dimensional case, Corollary (1) characterizes dispersive quantum systems within the class of completely positive Markovian quantum systems by a linear equation for its dissipation matrix:

$$
\sum_{i, j=1}^{N^{2}-1} a_{i j}\left(F_{j}^{*} H F_{i}-\frac{1}{2} F_{j}^{*} F_{i} H-\frac{1}{2} H F_{j}^{*} F_{i}\right)=0
$$

Just to emphasize, this equation is a necessary and sufficient condition for the system's Hamiltonian to belong to the kernel of its dissipator superoperator.

In the next section, I present a simple example of Markovian dispersive quantum system, showing that the class of such systems is non empty.

\section{Dispersive qubit}

The theoretical discussion of previous section does not follow the usual reasoning used in the modeling of physical systems. Instead, it just set the mathematical framework for modeling of quantum systems. In general, one associates to a quantum system its characteristic Hamiltonian and, in each specific situation that system is studied, its equation of motion is constructed taking into account the interaction with other systems and all relevant contributions to the system's dynamics due to its environment. This procedure is used when we want to model the system's behavior under specific conditions, or when we want to specify conditions for the system to behave according to some prescription. For completely positive Markovian quantum systems, one has to propose a Liouvillian and, to verify if that can be the generator of the system's quantum dynamical semigroup, perform one of the two following procedures:

i) Check the Hille-Yosida Theorem's condition (28);

ii) Solve the system's equation of motion 27, , built the dynamical semigroup and compute its generator.

Here I define dispersive qubit as a two-level quantum system having a special dynamics. The Hilbert space of this system is $\mathbb{C}^{2}$ with its usual structure of vector space and inner product. According with the general framework, observables are defined by self-adjoint operators and states are defined by density operators in $\mathbb{C}^{2}$. The system's equation of motion is the Markovian master equation 27 with Liouvillian having form (32) in terms of a Hamiltonian $H$ and a dissipator superoperator $\mathscr{D}$ :

$$
\frac{d}{d t} \rho(t)=-i[H, \rho(t)]+\mathscr{D}(\rho)
$$

The Hamiltonian has non-degenerated spectrum, with eigenvalues $E_{0}<E_{1}$. Using Dirac's notation, the corresponding normalized eigenvectors of the Hamiltonian are written as $\left|E_{0}\right\rangle$ and $\left|E_{1}\right\rangle$; using the ordered basis $\left\{\left|E_{1}\right\rangle,\left|E_{0}\right\rangle\right\}$, the space of linear operators $\mathscr{B}\left(\mathbb{C}^{2}\right)$ is identified with the space of $2 \times 2$ complex matrices $M_{2}(\mathbb{C})$; in particular, the Hamiltonian is given by the following diagonal matrix

$$
H=\left(\begin{array}{cc}
E_{1} & 0 \\
0 & E_{0}
\end{array}\right)
$$


I denote the identity matrix of $M_{2}(\mathbb{C})$ and Pauli matrices by

$$
\sigma_{0}:=\left(\begin{array}{ll}
1 & 0 \\
0 & 1
\end{array}\right), \sigma_{1}=\left(\begin{array}{ll}
0 & 1 \\
1 & 0
\end{array}\right), \sigma_{2}=\left(\begin{array}{cc}
0 & -i \\
i & 0
\end{array}\right), \sigma_{3}=\left(\begin{array}{cc}
1 & 0 \\
0 & -1
\end{array}\right)
$$

Pauli matrices with identity matrix divided by $\sqrt{2}$ form a basis for $M_{2}(\mathbb{C})$ which is orthonormal with respect to trace:

$$
\operatorname{tr}\left\{\frac{\sigma_{i}^{*}}{\sqrt{2}} \frac{\sigma_{j}}{\sqrt{2}}\right\}=\delta_{i j}, \forall i, j=0,1,2,3
$$

Therefore, one can use Pauli matrices to describe the system's Liouvillian and the dissipator superoperator, as prescribed by Theorem 3 and defined by (33):

$$
L \rho=-i[H, \rho]+\mathscr{D}(\rho)
$$

where

$$
H=\frac{E_{1}+E_{0}}{2} \sigma_{0}+\frac{E_{1}-E_{0}}{2} \sigma_{3}, \mathscr{D}(\rho)=\sum_{i, j=1}^{3} a_{i j}\left(\sigma_{i} \rho \sigma_{j}^{*}-\frac{1}{2}\left(\sigma_{j}^{*} \sigma_{i} \rho+\rho \sigma_{j}^{*} \sigma_{i}\right)\right)
$$

and $\left(a_{i j}\right)$ is the system's Kossakowski matrix. Defining

$$
\Delta:=E_{1}-E_{0}
$$

the system's dissipation operator 34 is given by:

$$
D_{H}:=\frac{\Delta}{2} \sum_{i, j=1}^{3} a_{i j}\left(\sigma_{j} \sigma_{3} \sigma_{i}-\frac{1}{2} \sigma_{j} \sigma_{i} \sigma_{3}-\frac{1}{2} \sigma_{3} \sigma_{j} \sigma_{i}\right)
$$

Finally, to complete the definition of dispersive qubit system I require that the dissipation operator is null:

$$
D_{H} \equiv 0
$$

This condition is an equation for Kossakowski matrix. Below, I deal with a special case.

\subsection{Special Dispersive Qubit}

From now on, I will deal with the following special case of dispersive qubit. For a fixed $\lambda>0$, called here dispersive parameter, I define the Kossakowski matrix:

$$
a_{i j}=\frac{1}{2} \lambda \delta_{i 3} \delta_{j 3}
$$

Finally, the explicit expression for the system's Liouvillian is

$$
L \rho=\frac{1}{2} \lambda\left(\sigma_{3} \rho \sigma_{3}-\rho\right)-i \frac{\Delta}{2}\left(\sigma_{3} \rho-\rho \sigma_{3}\right)
$$

In matricial terms:

$$
\rho=\left(\begin{array}{ll}
\rho_{11} & \rho_{12} \\
\rho_{21} & \rho_{22}
\end{array}\right) \leadsto L \rho=\left(\begin{array}{cc}
0 & -(\lambda+i \Delta) \rho_{12} \\
-(\lambda-i \Delta) \rho_{21} & 0
\end{array}\right)
$$


Time evolution. One can solve the equation of motion 46 and analyze the time evolution of states to get further details about what happens to the system as time goes on. Writing the density matrix for a generic time-dependent state

$$
\rho(t)=\left(\begin{array}{ll}
\rho_{11}(t) & \rho_{12}(t) \\
\rho_{21}(t) & \rho_{22}(t)
\end{array}\right), t \geq 0
$$

the system's equation of motion (46) becomes

$$
\left(\begin{array}{ll}
\dot{\rho}_{11}(t) & \dot{\rho}_{12}(t) \\
\dot{\rho}_{21}(t) & \dot{\rho}_{22}(t)
\end{array}\right)=\left(\begin{array}{cc}
0 & -(\lambda+i \Delta) \rho_{12}(t) \\
-(\lambda-i \Delta) \rho_{21}(t) & 0
\end{array}\right)
$$

The solution of this equation is

$$
\rho(t)=\left(\begin{array}{cc}
\rho_{11}(0) & e^{-(\lambda+i \Delta) t} \rho_{12}(0) \\
e^{-(\lambda-i \Delta) t} \rho_{21}(0) & \rho_{22}(0)
\end{array}\right), t \geq 0
$$

Under conditions which guarantee self-adjointness, positivity and trace one for $2 \times 2$ complex matrices, then the general form of the system's time-dependent states is:

$$
\rho(t)=\left(\begin{array}{cc}
a & b e^{-(\lambda+i \Delta) t} \\
\bar{b} e^{-(\lambda-i \Delta) t} & 1-a
\end{array}\right), t \geq 0
$$

where 11

$$
a \in \mathbb{R}, b \in \mathbb{C}, 0 \leq a \leq 1, a(1-a) \geq|b|^{2}
$$

The system's quantum dynamical semigroup follows from (61):

$$
\Gamma_{t} \rho=\left(\begin{array}{cc}
\rho_{11} & \rho_{12} e^{-(\lambda+i \Delta) t} \\
\rho_{21} e^{-(\lambda-i \Delta) t} & \rho_{22}
\end{array}\right), \forall \rho=\left(\begin{array}{ll}
\rho_{11} & \rho_{12} \\
\rho_{21} & \rho_{22}
\end{array}\right) \in \mathscr{L}(\mathscr{H})
$$

To verify the consistence of the model, we mention that $\Gamma_{t}$ is actually a continuous semigroup (in the sense of (19)) and its generator is $L$ :

$$
\lim _{t \downarrow 0} \frac{1}{t}\left(\Gamma_{t} \rho-\rho\right)=\lim _{t \downarrow 0}\left(\begin{array}{cc}
0 & \rho_{12} \frac{e^{-(\lambda+i \Delta) t}-1}{t} \\
\rho_{21} \frac{e^{-(\lambda-i \Delta) t}-1}{t} & 0
\end{array}\right)=L \rho, \forall \rho \in \mathscr{L}(\mathscr{H})
$$

Irreversibility. The condition of positiveness will be violated in 61 when $a<1$ and $|b|>0$ if one extrapolates this solution for times before

$$
t_{*}:=\ln \frac{1-a}{\lambda|b|}
$$

This fact suggests the model is non-time reversal invariant, since the quantum dynamical semigroup cannot be naturally extended to a one parameter group! Actually,

Proposition 2 The dispersive qubit is non-time reversal invariant.

\footnotetext{
11 We note that self-adjointness, positivity and trace one hold for all times $t \geq 0$ if and only if they hold for $t=0$.
} 
Proof It is sufficient to show that there is no idempotent solution to the time-reversing equation (6). So, assume that equation (6) has some solution $\Upsilon: \mathscr{T}(\mathscr{H}) \rightarrow \mathscr{T}(\mathscr{H})$ and write

$$
\Upsilon \rho=\left(\begin{array}{ll}
\gamma_{11}(\rho) & \gamma_{21}(\rho) \\
\gamma_{12}(\rho) & \gamma_{22}(\rho)
\end{array}\right), \forall \rho \in \mathscr{T}(\mathscr{H})
$$

For $\rho \in \mathscr{T}(\mathscr{H})$, denote $\rho_{*}:=\lim _{t \rightarrow \infty} \Gamma_{t} \rho$ as given by (71); then, from (6) it follows

$$
\Upsilon \rho=\lim _{t \rightarrow \infty}\left(\Gamma_{t} \Upsilon \Gamma_{t} \rho\right)=\lim _{t \rightarrow \infty}\left(\Gamma_{t} \Upsilon \lim _{t \rightarrow \infty} \Gamma_{t} \rho\right)=\left(\begin{array}{cc}
\gamma_{11}\left(\rho_{*}\right) & 0 \\
0 & \gamma_{22}\left(\rho_{*}\right)
\end{array}\right), \forall \rho \in \mathscr{T}(\mathscr{H})
$$

Therefore $\gamma_{12} \equiv 0 \equiv \gamma_{21}$ and this means $\Upsilon$ cannot be idempotent. This completes the proof.

Proposition 3 The dispersive qubit has pure states which evolve to mixed states. More precisely, an initial pure state remains pure if and only if it is one of the Hamiltonian's eigenstates.

Proof The density matrix for a pure state has the form

$$
\rho_{0}=\left(\begin{array}{cc}
|\lambda|^{2} & \lambda \bar{\beta} \\
\bar{\lambda} \beta & |\beta|^{2}
\end{array}\right), \lambda, \beta \in \mathbb{C},|\lambda|^{2}+|\beta|^{2}=1
$$

I note that $\operatorname{det} \rho_{0}=0$ and this condition is necessary for any density matrix representing a pure state.

The state $\rho_{0}$ is one of the two eigenstates of Hamiltonian if and only if $\lambda=0$ or $\beta=0$; from (61), those states are pure and from (61) remain constant (and pure).

However, any initially pure state $\rho(0)=\rho_{0}$ with $\lambda \neq 0$ and $\beta \neq 0$ evolves to impure states, since

$$
\operatorname{det} \rho(t)=\operatorname{det}\left(\begin{array}{cc}
|\lambda|^{2} & \lambda \bar{\beta} e^{-(\lambda+i \Delta) t} \\
\bar{\lambda} \beta e^{-(\lambda-i \Delta) t} & |\beta|^{2}
\end{array}\right)=|\lambda|^{2}|\beta|^{2}\left(1-e^{-2 \lambda t}\right) \neq 0, \forall t>0
$$

The system's stationary states are defined by the limit

$$
\lim _{t \rightarrow \infty} \Gamma_{t} \rho, \rho \in \mathscr{S}(\mathscr{H})
$$

They are explicitly

$$
\rho_{a}:=\left(\begin{array}{cc}
a & 0 \\
0 & 1-a
\end{array}\right), 0 \leq a \leq 1
$$

The von Neumann entropy [24, p.510] of the state [61] is given by:

$$
\begin{aligned}
S[\rho(t)]= & -\operatorname{tr}\{\rho(t) \log \rho(t)\} \\
= & \log 2-a \log \left(1+\sqrt{1-4\left(a(1-a)-|b|^{2} e^{-2 \lambda t}\right)}\right)+ \\
& -(1-a) \log \left(1-\sqrt{1-4\left(a(1-a)-|b|^{2} e^{-2 \lambda t}\right)}\right)
\end{aligned}
$$


As one can verify, $S[\rho(t)]$ increases with time when $b \neq 0$ and

$\lim _{t \rightarrow \infty} S[\rho(t)]=\log 2-a \log (1+\sqrt{1-4 a(1-a)})-(1-a) \log (1-\sqrt{1-4 a(1-a)})$

For the special case $a=1 / 2$, this limit reaches the entropy's maximum value [24 p.513]:

$$
\lim _{t \rightarrow \infty} S[\rho(t)]=\log 2
$$

\subsubsection{General observables}

Let $X$ be an observable of the Dispersive Qubit, i.e., a self-adjoint operator in $\mathbb{C}^{2}$. It has two eigenvalues (which can be equal) $x_{1} \geq x_{2}$ and corresponding orthonormal eigenvectors $\left|x_{1}\right\rangle$ and $\left|x_{2}\right\rangle$ :

$$
X\left|x_{j}\right\rangle=x_{j}\left|x_{j}\right\rangle,\left\langle x_{i} \mid x_{j}\right\rangle=\delta_{i j} ; i, j=1,2
$$

From above conditions and with an eventual redefinition of $\left|x_{1}\right\rangle$ and $\left|x_{2}\right\rangle$, it follows that there exists $\theta \in[0, \pi / 2]$ such that

$$
\left\{\begin{array}{l}
\left|x_{1}\right\rangle=\cos \theta|+\rangle+\sin \theta|-\rangle \\
\left|x_{2}\right\rangle=-\sin \theta|+\rangle+\cos \theta|-\rangle
\end{array}\right.
$$

In the basis $\left\{\left|E_{1}\right\rangle,\left|E_{0}\right\rangle\right\}$, we have

$$
\left|x_{1}\right\rangle=\left(\begin{array}{c}
\cos \theta \\
\sin \theta
\end{array}\right),\left|x_{2}\right\rangle=\left(\begin{array}{c}
-\sin \theta \\
\cos \theta
\end{array}\right)
$$

and

$$
X=\left(\begin{array}{c}
x_{1} \cos ^{2} \theta+x_{2} \sin ^{2} \theta\left(x_{1}-x_{2}\right) \sin \theta \cos \theta \\
\left(x_{1}-x_{2}\right) \sin \theta \cos \theta x_{1} \sin ^{2} \theta+x_{2} \cos ^{2} \theta
\end{array}\right)
$$

I remark that the observable $X$ is not compatible with the Hamiltonian $H$ when $\theta \neq 0$ and $\theta \neq \pi / 2$, because they cannot be simultaneously diagonalized.

Time evolution of eigenstates of $X$. If the initial state of the system is that corresponding to the eigenstate $\left|x_{1}\right\rangle$ of $X$

$$
\rho_{x_{1}}(0)=\left|x_{1}\right\rangle\left\langle x_{1}\right|=\left(\begin{array}{cc}
\cos ^{2} \theta & \cos \theta \sin \theta \\
\cos \theta \sin \theta & \sin ^{2} \theta
\end{array}\right)
$$

Then, according with 60 the time-dependent state is given by

$$
\rho_{x_{1}}(t)=\left(\begin{array}{cc}
\cos ^{2} \theta & e^{-(\lambda+i \Delta) t} \cos \theta \sin \theta \\
e^{-(\lambda-i \Delta) t} \cos \theta \sin \theta & \sin ^{2} \theta
\end{array}\right), t \geq 0
$$

If $\theta \neq 0$ and $\theta \neq \pi / 2$, than the state $\rho_{x_{1}}(t)$ is a mixture for all $t>0$, since it cannot be put in the form (68). In particular, the system evolves from the pure state $\left|x_{1}\right\rangle\left\langle x_{1}\right|$ to the mixture

$$
\lim _{t \rightarrow \infty} \rho_{x_{1}}(t)=\cos ^{2} \theta\left|E_{1}\right\rangle\left\langle E_{1}\left|+\sin ^{2} \theta\right| E_{0}\right\rangle\left\langle E_{0}\right|
$$


Expectation values of $X$. The expectation value of $X$ w.r.t. a generic time-dependent state (61) is

$$
\begin{aligned}
\langle X \mid \rho(t)\rangle= & a\left[x_{1} \cos ^{2} \theta+x_{2} \sin ^{2} \theta\right]+b e^{-(\lambda+i \Delta) t}\left[\left(x_{1}-x_{2}\right) \sin \theta \cos \theta\right]+ \\
& +\bar{b} e^{-(\lambda-i \Delta) t}\left(x_{1}-x_{2}\right) \sin \theta \cos \theta+(1-a)\left[x_{1} \sin ^{2} \theta+x_{2} \cos ^{2} \theta\right]
\end{aligned}
$$

In particular, the stationary expectation value of $X$ is

$\lim _{t \rightarrow \infty}\langle X \mid \rho(t)\rangle=\left\langle X \mid \lim _{t \rightarrow \infty} \rho(t)\right\rangle=\left(a \cos ^{2} \theta+(1-a) \sin ^{2} \theta\right) x_{1}+\left(a \sin ^{2} \theta+(1-a) \cos ^{2} \theta\right) x_{2}$

Combining the above formulas, we get the expectation value of $X$ w.r.t. the timedependent state which started as the $X$ 's vector-state $\left|x_{1}\right\rangle$ :

$\left\langle X \mid \rho_{x_{1}}(t)\right\rangle=\left(\cos ^{4} \theta+\sin ^{4} \theta\right) x_{1}+2 \cos ^{2} \theta \sin ^{2} \theta x_{2}+e^{-\lambda} \sin ^{2} \theta \cos ^{2} \theta \cos (i \Delta t)\left(x_{1}-x_{2}\right)$

and

$$
\lim _{t \rightarrow \infty}\langle X \mid \rho(t)\rangle=\left\langle X \mid \lim _{t \rightarrow \infty} \rho(t)\right\rangle=\left(\cos ^{4} \theta+\sin ^{4} \theta\right) x_{1}+2 \cos ^{2} \theta \sin ^{2} \theta x_{2}
$$

Probabilities of transition and of surviving. The transition probability from the $X$ 's vector-state $\left|x_{1}\right\rangle$ to the $X$ 's vector-state $\left|x_{2}\right\rangle$ after a time $t \geq 0$ is given by the expectation value of the projection operator $\left|x_{2}\right\rangle\left\langle x_{2}\right|$ :

$$
P\left(x_{1} \rightarrow x_{2} ; t\right)=\operatorname{tr}\left(\left|x_{2}\right\rangle\left\langle x_{2}\right| \rho_{x_{1}}(t)\right)
$$

Explicitly:

$$
\begin{aligned}
P\left(x_{1} \rightarrow x_{2} ; t\right) & =\left\langle E_{1} \mid x_{2}\right\rangle\left\langle x_{2}\left|\rho_{x_{1}}(t)\right| E_{1}\right\rangle+\left\langle E_{0} \mid x_{2}\right\rangle\left\langle x_{2}\left|\rho_{x_{1}}(t)\right| E_{0}\right\rangle \\
& =2\left[1-e^{-\lambda t} \cos (\Delta t)\right] \cos ^{2} \theta \sin ^{2} \theta
\end{aligned}
$$

The surviving probability of the $X$ 's vector-state $\left|x_{1}\right\rangle$ after a time $t \geq 0$ is given by the expectation value of the projection operator $\left|x_{1}\right\rangle\left\langle x_{1}\right|$ :

$$
P\left(x_{1} \rightarrow x_{1} ; t\right)=\operatorname{tr}\left(\left|x_{1}\right\rangle\left\langle x_{1}\right| \rho_{x_{1}}(t)\right)
$$

Explicitly:

$$
\begin{aligned}
P\left(x_{1} \rightarrow x_{1} ; t\right) & =\left\langle E_{1} \mid x_{1}\right\rangle\left\langle x_{1}\left|\rho_{x_{1}}(t)\right| E_{1}\right\rangle+\left\langle E_{0} \mid x_{1}\right\rangle\left\langle x_{1}\left|\rho_{x_{1}}(t)\right| E_{0}\right\rangle \\
& =\cos ^{4} \theta+\sin ^{4} \theta+2 e^{-\lambda t} \cos (\Delta t) \cos ^{2} \theta \sin ^{2} \theta
\end{aligned}
$$

One can easily verify that

$$
0 \leq P\left(x_{1} \rightarrow x_{2} ; t\right), P\left(x_{1} \rightarrow x_{1} ; t\right) \leq 1 ; P\left(x_{1} \rightarrow x_{2} ; t\right)+P\left(x_{1} \rightarrow x_{1} ; t\right)=1, \forall t \geq 0
$$

For later use, I rewrite above formulas:

$$
P\left(x_{1} \rightarrow x_{2} ; t\right)=\left[\frac{1}{2}-e^{-\lambda t}\left(\frac{1}{2}-\sin ^{2}\left(\frac{\Delta}{2} t\right)\right)\right] \sin ^{2}(2 \theta)
$$




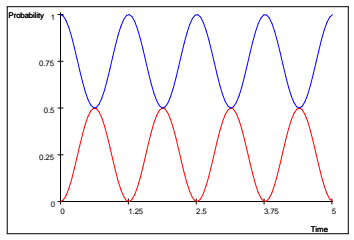

Fig. 1 Transition and surviving probabilities for $\Delta=5, \theta=\pi / 8, \lambda=0$

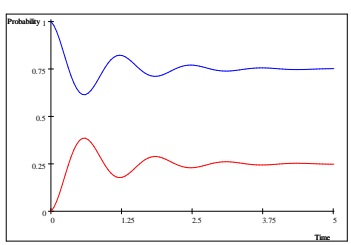

Fig. 2 Transition and surviving probabilities for $\Delta=5, \theta=\pi / 8, \lambda=1$

$$
P\left(x_{1} \rightarrow x_{1} ; t\right)=1-\left[\frac{1}{2}-e^{-\lambda t}\left(\frac{1}{2}-\sin ^{2}\left(\frac{\Delta}{2} t\right)\right)\right] \sin ^{2}(2 \theta)
$$

I remark the role of the dispersive parameter $\lambda$ of dispersive qubit: it changes the initially time-dependent superposition of the states of $X$ to a final (assymptotic) time-indepented mixture of them!

To ilustrate the time evolution of the special dispersive qubit, I plot in the fig 1 and fig 2 the graphs of the time-dependent transition and surviving probabilities corresponding to $\Delta=5$ and $\theta=\pi / 8$ for $\lambda=0$ and $\lambda=1$.

Is there any application of the previous concepts and developments? Maybe...

\subsubsection{Remark on the Kossakowski matrix}

The following proposition shows that the choice (57) for the Kossakowski matrix cannot be otherwise:

Proposition 4 The dispersive qubit's dissipator operator (53) is zero if and only if its dissipation matrix $\left(a_{i j}\right)$ satisfies

$$
a_{i j}=\beta \delta_{i 3} \delta_{j 3}, \beta \geq 0
$$

Proof We have to find all solutions to the equation for Kossakowski matrix's coeffcients

$$
\frac{1}{2} \Delta \sum_{i, j=1}^{3} a_{i j}\left(\sigma_{j} \sigma_{3} \sigma_{i}-\frac{1}{2} \sigma_{j} \sigma_{i} \sigma_{3}-\frac{1}{2} \sigma_{3} \sigma_{j} \sigma_{i}\right)=0
$$

subject to the conditions which guarantee self-adjointness

$$
\text { (s.a) } \bar{a}_{i j}=a_{j i}, \forall i, j=1,2,3
$$


and one of the following two sets of conditions which guarantee positive-semi definiteness for $3 \times 3$ matrices:

$$
\begin{aligned}
& (i): \operatorname{tr}\left(a_{i j}\right)>0, \operatorname{det}\left(a_{i j}\right)>0, \frac{1}{2} \operatorname{tr}\left(a_{i j}\right)\left(\left(\operatorname{tr}\left(a_{i j}\right)\right)^{2}-\operatorname{tr}\left(\left(a_{i j}\right)^{2}\right)\right)>\operatorname{det}\left(a_{i j}\right) \\
& (i i): \operatorname{tr}\left(a_{i j}\right)>0, \operatorname{det}\left(a_{i j}\right)=0, \frac{1}{2} \operatorname{tr}\left(a_{i j}\right)\left(\left(\operatorname{tr}\left(a_{i j}\right)\right)^{2}-\operatorname{tr}\left(\left(a_{i j}\right)^{2}\right)\right) \geq 0
\end{aligned}
$$

For $\beta>0$, the matrix $\left(a_{i j}=\beta \delta_{i 3} \delta_{j 3}\right)$ is positive since it obviously self-adjoint and has only non-negative eigenvalues (namely, 0 and $\beta \sqrt{12}$. by direct verification we see it satisfies equation (85):

$$
\frac{\Delta \beta}{2}\left(\sigma_{3} \sigma_{3} \sigma_{3}-\frac{1}{2} \sigma_{3} \sigma_{3} \sigma_{3}-\frac{1}{2} \sigma_{3} \sigma_{3} \sigma_{3}\right)=0
$$

Now, it is very tedious to write down the calculations to get all solutions for the above matrix equation (85) subject to (86) with one of the above two positivity conditions 87); besides, such calculations are not directly relevant to the purposes of this paper; so I omit those here.

\section{Dispersive model for neutrino oscillation}

Neutrinos are neutral leptons which occur in one out of three flavors (related to the others three charged leptons: electron, muon and tauon). They have very tiny masses and interact extremely feebly, being sensible to weak interaction and gravity only. Those characteristics mean that neutrinos are "quasi free" particles - or more precisely, they propagate almost unperturbed during the (eventually large) time lapse between their production and detection. So, it is natural to pay attention in neutrinos in the search to find a system combining both "isolatedness" and non-time reversal invariance.

The phenomenology of neutrinos is not completely theoretically understood [25]. Actually, the Standard Model of Particle Physics and what must extend/replace it are at stake [26]. Specifically, the phenomenon of neutrino oscillation (defined as the dynamic change of flavor) is a compelling evidence that those particles have masses different from zero, an explicit contradiction with the Standard Model [27], [28], [29],[30], [31]. The theoretical mechanism explaining neutrino oscillation was first devised by Pontecorvo [27] and [28] around the 1960s; the first experimental evidence of neutrino oscillation was obtained in the Super-Kamiokande experiment in 1998 [29], and since them several experiments have been realized around the world to measure the parameters associated with neutrinos [30], [31, pp.114-183], [33], [32].

Further, there are empirical evidences [34] as well as theoretical reasons [31] for the existence of (at least) one more neutrino flavor (besides the three standard flavors), called sterile neutrino. Presumably, sterile neutrino is a hight-handed particle which mix itself with the other neutrino species and interacts only through gravity what makes it be a very ghostly particle. From the original proposal to understand

\footnotetext{
12 Equivalently, $\left(a_{i j}=\beta \delta_{i 3} \delta_{j 3}\right)$ satisfies conditions 86 and 87 .ii).
} 
unexpected data concerning neutrino oscillation, some speculate that sterile neutrino can also explain the large disparity of leptons masses as well as be the reason behind the matter-antimatter asymmetry and, further, that it can be the main missing ingredient of the Universe (as the predominant component of dark matter) [31, pp.114-183], [35]. The possibility of "new physics beyond Standard Model" is enlarged by unusual ideas to describe neutrino dynamics and mixing of flavors: non-standard interactions [36] and non-unitary time evolution of flavor-states [37], [38].

As long as there still are deep open questions about neutrinos, I think it is opportune investigate the possibility that the dynamics of neutrinos can be "dispersive", i.e., intrinsically non-time reversal invariant - more precisely: that the time evolution of neutrinos is non-time reversal invariant even when they propagate isolately (in vacuum).

Specifically, I describe below the neutrino oscillation between two flavor ${ }^{13}$ throughout a phenomenological approach similar to the original ones [27] [28], called theory of massive and mixed neutrino in the monograph [39] - my basic reference ${ }^{14}$ In this theory, neutrino flavor states are superpositions of eigenstates of the relativistic mass operator [39, Chapter 4]; in the quantum mechanical approximation, the theory reduces to the case described in the subsection 3.1 .1

Dispersive theory of massive and mixed neutrino. The oscillation between two neutrino flavors is described by a two-level quantum system, identical to the dispersive quibit defined in the previous section. The flavor states, denoted by $\left|\bar{v}_{e}\right\rangle$ and $\left|\bar{v}_{\mu}\right\rangle$, are superpositions of the Hamiltonian's eigenvectors, denoted by $\left|E_{v_{e}}\right\rangle$ and $\left|E_{v_{\mu}}\right\rangle$ through relation (77), where $\theta$ is called the mixing angle [39, pp.107-108]. Here ${ }^{15}$ I assume the dynamics is given by Lindblad's equation (46) with Hamiltonian and dissipator superoperator (3) and Kossakowski matrix (55) with a dispersive parameter $\lambda(56)$ :

$$
\frac{d \rho}{d t}=\frac{1}{2}\left(\lambda \sigma_{3} \rho \sigma_{3}-\rho\right)-i \frac{\Delta}{2}\left(\sigma_{3} \rho-\rho \sigma_{3}\right)
$$

where (as in subsection 3.1.1

$$
\Delta=E_{v_{\mu}}-E_{v_{e}}
$$

Since neutrinos are produced with speed near the light velocity $(c=1)$, their energies must be given by the relativistic formula which combines mass and momentum [39, p.99]:

$$
E_{\chi}=\sqrt{p_{\chi}^{2}+m_{\chi}^{2}}, \chi=v_{e}, v_{\mu}
$$

I assume the following conditions [39, p.105]:

\footnotetext{
13 The oscillation between two neutrino flavors can be applied very well to the solar neutrinos, since for them only the oscillation of $v_{e}$ and $v_{\mu}$ is relevant

14 Reference [40] also develops a more rigorous treatment which justifies the probabilities of transition and surviving we find below (but only in the dispersiveless case, $\lambda=0$ ).

15 In the standard approach, one assumes that neutrino dynamics is given by Schrödinger equation with Hamiltonian $H$ [39. p.99].
} 
i) The masses of neutrinos are small compared to the momenta they are produced:

$$
\frac{m_{\chi}}{p_{\chi}} \ll 1, \chi=v_{e}, v_{\mu}
$$

ii) The momenta of neutrinos are approximately equal:

$$
E:=p_{v_{e}} \simeq p_{v_{\mu}}
$$

With above assumptions, it holds the approximations

$$
E_{\chi} \simeq p_{\chi}+\frac{m_{\chi}^{2}}{2 p_{\chi}^{2}} \simeq E+\frac{m_{\chi}^{2}}{2 E^{2}}, \chi=v_{e}, v_{\mu}
$$

and

$$
\Delta=E_{v_{\mu}}-E_{v_{e}} \simeq \frac{m_{v_{\mu}}^{2}-m_{v_{e}}^{2}}{2 E}
$$

The time lapse between the production and the detection of the neutrinos is approximately given in terms of the distance $L$ between the source and the detector by $(c=1)$

$$
t=L
$$

Finally, substituting 89 and 90 in the formulas for the probability of transition 82) and surviving (83) it follows with the insertion of constants $c$ and $\hbar$ :

$$
P\left(\bar{v}_{e} \rightarrow \bar{v}_{\mu} ; L, E, \lambda\right)=\left[\frac{1}{2}-e^{-\lambda L / c}\left(\frac{1}{2}-\sin ^{2}\left(\frac{m_{1}^{2}-m_{0}^{2}}{4 \hbar / c^{3}} \frac{L}{E}\right)\right)\right] \sin ^{2}(2 \theta)
$$

and

$$
P\left(\bar{v}_{e} \rightarrow \bar{v}_{e} ; L, E, \lambda\right)=1-\left[\frac{1}{2}-e^{-\lambda L / c}\left(\frac{1}{2}-\sin ^{2}\left(\frac{m_{1}^{2}-m_{0}^{2}}{4 \hbar / c^{3}} \frac{L}{E}\right)\right)\right] \sin ^{2}(2 \theta)
$$

In the case $\lambda=0$, these formulas are reduced to the standard ones [39] pp.108109].

Assuming $\lambda=0$, experimental data from KamLAND for the oscillation between antineutrino-eletron $\bar{v}_{e}$ to the antineutrino-muon $\bar{v}_{\mu}$ gives the following values for physical constants [30]:

$$
m_{v_{\mu}}^{2}-m_{v_{e}}^{2}=7.9_{-0.5}^{+0.6} \times 10^{-5} e V^{2}, \tan ^{2} \theta=0.40_{-0.7}^{+0.10}
$$

Finally, I leave to the experts the analysis of experimental data taking into account the dispersive parameter ${ }^{16}$ is it possible that the data can eventually corroborate the hypothesis that neutrino dynamics have a dispersive parameter different from zero?

\footnotetext{
16 Since the subject of this section is complex and out of my expertise, my intention is modest: illustrate the application of dispersive quantum system and incite further researches on the subject.
} 


\section{Conclusions}

To summing up, the effect of non-unitary time evolution in quantum systems can be threefold: dissipation/gain (which means variation of system's energy), impurification (which means time evolution from pure states to mixed states, what is measured by the systems' entropy) and decoherence (which happens only to composed systems and was not discussed here). In general, those phenomena are related to non-time reversal invariance and occur typically in open systems - the environment being the system's partner to the exchanges of matter, energy, momenta and information (entropy). That is our expectation for completely positive Markovian quantum systems with non-zero dissipator superoperator (which means a deviation from unitary time evolution); however, for the class of dispersive quantum systems, time evolution implies impurification even when the systems are isolated.

Surely, I don't know if the concept of dispersive quantum system can help us deepen our understanding about time reversal invariance and the meaning of irreversibility. Nevertheless, the existence of an actual elementary dispersive quantum systems would be remarkable, since for them the non-time reversal invariance (and the property of the system's entropy be non-decreasing with time) cannot have a statistical meaning.

If one does not forget the essential difference concerning statistical interpretation, it can be useful to think about dispersive quantum systems as the quantum analogs of classical isolated thermodynamical systems (such as a low density gas in free expansion) because both can be isolated and non-time reversal invariant.

I glimpse some developments to be done from what was presented here:

i) The characterization of dispersive quantum systems with infinite degrees of freedom;

ii) The study of decoherence in the context of dispersive quantum systems;

iii) Improving the modeling of neutrino oscillation.

iv) The building of new models and the search to get, in the laboratory, instances of dispersive quantum systems 17

Finally, the issue deserves further theoretical as well as experimental researches, if not due to the possibility to describe actual physical systems (like neutrinos), at least because we ignore answers to this simple question: If dispersive quantum systems cannot exist in nature (even approximately), are there physical principles forbidding them to exist?

\section{A Completely positive quantum dynamical semigroups}

Here, I present the definition of a completely positive quantum dynamical semigroup following [5] (using a different notation). Denote by $M_{m}(\mathbb{C})$ the $\mathrm{C}^{*}$-algebra of $m \times m$ complex matrices with identity $I_{m}$, for any positive integer $m$.

\footnotetext{
17 Perhaps, dispersive quantum systems can be manipulated in the laboratory by adjusting the environment into which a quantum system is inserted so that it behaves as if it was isolated, up to a satisfactory degree.
} 
Definition 5 (Completely Positive Map) Let $\mathfrak{A}$ and $\mathfrak{B}$ be $\mathrm{C}^{*}$-algebras. An operator $f: \mathfrak{A} \rightarrow \mathfrak{B}$ is said to be completely positive if and only if for all positive integer $m$ the following map is positive:

$$
f^{(m)}:=f \otimes I_{m}: \mathfrak{A} \otimes M(m) \rightarrow \mathfrak{B} \otimes M(m)
$$

Definition 6 (Dual Map) The dual of an operator $\Phi: \mathscr{T}(\mathscr{H}) \rightarrow \mathscr{T}(\mathscr{H})$ is the operator $\Phi^{*}: \mathscr{B}(\mathscr{H}) \rightarrow$ $\mathscr{B}(\mathscr{H})$ defined by the following equation:

$$
\operatorname{tr}\left(\sigma \Phi^{*}(B)\right)=\operatorname{tr}(B \Phi(\sigma)), \forall \sigma \in \mathscr{T}(\mathscr{H}), \forall B \in(\mathscr{B}(\mathscr{H}))
$$

Definition 7 (Dual Quantum Dynamical Semigroup) The dual of the quantum dynamical semigroup $\Gamma_{t \geq 0}: \mathscr{T}(\mathscr{H}) \rightarrow \mathscr{T}(\mathscr{H})$ is the one-parameter semigroup of dual maps:

$$
0 \leq t \mapsto \Gamma_{t}^{*}: \mathscr{B}(\mathscr{H}) \rightarrow \mathscr{B}(\mathscr{H})
$$

Definition 8 (Completely Positive Quantum Dynamical Semigroup) The quantum dynamical semigroup $\Gamma_{t \geq 0}$ is said to be completely positive if and only if for all $t \geq 0$ the dual operator $\Gamma_{t}^{*}$ is completely positive.

Remark 9 In Quantum Mechanics, the dual quantum dynamical semigroup corresponds to the Heisenberg picture for time evolution.

\section{References}

1. C. Rovelli, "Forget time": Essay written for the FQXi contest on the Nature of Time, Found. Phys. 41 (2011) 1475-1490.

2. H.D. Zeh, The Physical Basis of the Direction of Time - 5th edition, Springer, Berlin-Heidelberg, 2010.

3. H.-P. Breuer, F. Petruccione, The Theory of Open Quantum Systems, Oxford University Press, Oxford, 2003

4. G.E. Crooks, Quantum Operation Time Reversal. Phys. Rev. A 77 (2008) 034101.

5. V. Gorini, A. Kossakowski, E.C.G. Sudarshan, Completely positive dynamical semigroups of N-level systems, J.Math.Phys. 17 (1976) 821-825.

6. G. Lindblad, On the generator of quantum dynamical semigroups, Commun. Math. Phys 48 (1976) $119-130$.

7. J. Mehra, E.C.G. Sudarshan, Nuovo Cimento 11B (1972) 215-256.

8. A. Kossakowski, Rep. Math. Phys. 3 (1972) 247-274.

9. R.S. Ingarden, A. Kossakowski, Ann. Phys. 89 (1975) 451-485

10. J. Uffink, Three concepts of irreversibility and three versions of the second law in Time and History, in: F. Stadler and M. Stoelzner (eds) Heussenstamm, Proceedings of the 28. International Ludwig Wittgenstein Symposium, Ontos Verlag(2006): 275-288.

11. M. Aiello, M. Castagnino, L. Lombardi, The Arrow of Time: From Universe Time-Asymmetry to Local Irreversible Processes, Found. Phys. 38 (2008) 257-292.

12. L.D. Landau, E.M. Lifshitz, Statistical Physics - 3th edition, Pergamon Press, New York, 1977.

13. L.D. Landau, E.M. Lifshitz, Quantum Mechanics: non-relativisitic theory - 3th edition, Pergamon Press, New York, 1977.

14. E.B. Davies, Markovian Master Equations, Commun. Math. Phys. 39(2) (1974) 91-110.

15. K. Kraus, States, Effects and Operations (Lecture Notes on Physics, vol. 190), Springer-Verlag, Berlin, 1983.

16. P. Pechukas, Reduced dynamics need not be completely positive, Physical Review Letters 73(8) (1994) 1060-1062.

17. F. Ticozzi, M. Pavon, On time-reversal and space-time harmonic processes for Markovian quantum channels, Quantum Inf. Process 9 (2010) 551-574.

18. H. Yu, J. Zhang, Understanding Hawking radiation in the framework of open quantum systems, Phys. Rev. D 77 (2008) 024031.

19. H. Yu, Open Quantum System Approach to the Gibbons-Hawking Effect of de Sitter Space-Time, Phys. Rev. Let. 106 (2011) 061101. 
20. F. Benatti, R. Floreanini, Entanglement generation in uniformly accelerating atoms: Reexamination of the Unhru effect. Phys. Rev. A 70 (2004) 012112.

21. M. Reed, B. Simon, B.: Methods of Modern Mathematical Physics I: Functional Analysis, Revised and Enlarged Edition, Academic Press, New York, 1980.

22. W. Rudin, Functional Analysis, 2th edition, McGraw-Hill, Boston 1991.

23. K. Yosida, Functional Analysis, 6th edition, Springer-Verlag, Berlin, 1980.

24. I.L. Chuang, M.A. Nielsen, Quantum Computation and Quantum Information, Cambridge University Press, Cambridge, 2000.

25. E. Kh. Akhmedov, A. Yu. Smirnov, Paradoxes of Neutrino Oscillations. Physics of Atomic Nuclei, 72(8) (2009) 1363-1381.

26. J. Holeczek, J. Kisiel, J. Syska, M. Zraleka, Searching for new physics in future neutrino factory experiments, Eur. Phys. J. C 52 (2007) 905-917.

27. B. Pontecorvo, Mesonium and antimesonium, Sov. Phys. JETP 6 (2957) 429; Inverse beta processes and nonconservation of lepton charge, ibid 7 (1958) 172; Neutrino experiments and the question of leptonic-charge conservation, ibid, 26 (1968) 984.

28. Z. Maki, M. Nakagawa, S. Sakata, Prog. Theor. Phys. 28 (1962) 870.

29. Y. Fukuda et al. (Super-Kamiokande Collaboration): Evidence for oscillation of atmospheric neutrinos, Phys. Rev. Lett. 81 (1998) 1562. [hep-ex/9807003]

30. T. Araki et al. (KamLAND Collaboration), Measurement of Neutrino Oscillation with KamLAND: Evidence of Spectral Distortion, Physical Review Letters 94 (2005) 081801.

31. N. Nakamura et al. (Particle Data Group), Review of Particle Physics, J. Phys. G: Nucl. Part. Phys. 37 (2010) 075021.

32. G. Mention et al., Reaction antineutrino anomaly, Phys. Rev. D, 83 (2011) 073006.

33. B. Aharmin et al. (SNO Collaboration), Phys. Rev. C 72 (2005) 055502.

34. A. Aguilar et al. (LSND Collaboration), Evidence for neutrino oscillations from the observation of $\bar{v}_{e}$ e appearance in a $\bar{v}_{\mu}$ beam. Phys. Rev. D 64 (2001) 112007; Event Excess in the MiniBooNE Search for $\bar{v}_{\mu} \rightarrow \bar{v}_{e}$ Oscillations, Phys. Rev. Lett. 105 (2010) 181801.

35. R. Jimenez, Cosmological insights into fundamental physics, Fortschr. Phys. 59, (No.7-8) (2011) 602 $-617$.

36. P. Coloma, A. Donini, J. López-Pavónd, H. Minakatae, Non-standard interactions at a neutrino factory: correlations and CP violation. J. High Energy Phys. 08 (2011) 036.

37. S. Antusch, C. Biggio, E. Fernandez-Martinez, M.B. Gavela, J. López-Pavónd, Unitarity of leptonic mixing matrix. J. High Energy Phys. 10 (2006) 084.

38. S. Antusch, M. Blennow, E. Fernandez-Martinez, T. Ota, New physics searches at near detectors of neutrino oscillation experiments, J. High Energy Phys. 06 (2010) 068.

39. S. Bilenky, Introduction to Physics of Massive and Mixed Neutrinos (Lecture Notes in Physics 817), Springer, Berlin-Heidelberg, 2010.

40. H.J. Lipkin, Quantum theory of neutrino oscillations for pedestrians: simple answers to confusing questions, Physics Letters B 642 (2006) 366-371. 